\title{
Subacute sensory neuropathy with remission: an association with lymphoma
}

\author{
HJ SAGAR, DJ READ \\ From the Department of Neurology, Radcliffe Infirmary, Oxford
}

SUMMARY A case of subacute sensory neuropathy associated with Hodgkin's disease improved as the tumour was treated. The poor prognosis usually associated with this neuropathy may be attributable to its more common association with untreatable tumours.

Subacute sensory neuropathy is recognised as a complication of carcinoma and is said to carry a poor prognosis. ${ }^{1}$ We report a case of this syndrome associated with lymphoma in which successful treatment of the tumour was associated with considerable improvement in the neuropathy.

\section{Case report}

A 46-year-old woman was investigated for low back pain which was exacerbated by her taking even small quantities of alcohol. Her blood count showed a haemoglobin of $13.4 \mathrm{~g} / \mathrm{dl}$ with a slight leukocytosis $\left(12.00 \times 10^{9} / \mathrm{l}\right)$ and an elevated plasma viscosity $(2 \cdot 16$; normal up to $1 \cdot 72)$. There were elevated serum concentrations of IgG (21.6 g/l; normal 7-19) and IgA (7.95 g/l; normal 0.9-4.5) but the IgM concentration was normal $(2 \cdot 18 \mathrm{~g} / 1$; normal $0 \cdot 55-2 \cdot 20)$. Bone marrow histology was normal. No abnormality was seen on lumbar myelography or barium enema examination.

Two years later she also developed ataxia of gait which progressed until three weeks later she was completely unable to walk. Ten days after the onset of the ataxia, she developed painful paraesthesiae and loss of tactile sensibility over the right side of the face and in a glove and stozking distribution in the limbs. Neurological examination revealed moderate bilateral weakness of hip flexion and extension but otherwise normal power; markedly impaired superficial sensation over the distribution of the second and third divisions of the right trigeminal nerve and bilaterally below C3; absent proprioception in the limbs; absent vibration sense below the xiphisternum; and complete areflexia. She could stand but was unable to walk because of profound ataxia. Limb movements were clumsy but there was no tremor. She

Address for reprint requests: Dr HJ Sagar, Department of Neurology, Radcliffe Infirmary, Oxford, UK.

Received 23 August 1981

Accepted 25 October 1981 did not have nystagmus. General medical examination showed right inguinal and para-aortic lymphadenopathy. Serum protein electrophoresis showed a diffuse increase in gamma-globulins due to a raised IgG concentration $(23 \mathrm{~g} / \mathrm{l})$. Examination of the CSF showed a markedly raised protein concentration $(3.0 \mathrm{~g} / \mathrm{l})$ and a relative increase in $\mathrm{IgG}$ concentration $(1.006 \mathrm{~g} / \mathrm{l})$ but a normal cell count, glucose concentration and viral antibody titres. Chest radiology and abdominal lymphangiography showed paratracheal, pelvic and abdominal lymphadenopathy which was shown by inguinal node biopsy and laparotomy to be associated with stage $3 \mathrm{~b}$ mixed cellularity Hodgkin's disease. Electrophysiological examination showed partial denervation of all hand and foot muscles. The maximum conduction velocity was $25 \mathrm{~m} / \mathrm{s}$ in the leg but was normal in the arm. There were no recordable sensory potentials in the upper limb nerves. Extensive investigation failed to reveal any cause for the peripheral neuropathy other than a remote effect of the lymphoma.

Her neurological condition remained unchanged for a further four weeks. Treatment was then begun with prednisolone $60 \mathrm{mg}$ daily and pulses of procarbazine, nitrogen mustard and vinblastine. She improved rapidly so that after four days she was walking with the aid of a frame and no longer suffered from paraesthesiae. Examination then showed regression of the distal sensory loss to the elbows in the arms and to the lower third of the thighs in the legs; there was no weakness. By one month later, she could manage her housework, climb stairs and bath without aids. The improvement was maintained through five further pulses of cytotoxic therapy over the next eight months except for two minor relapses associated with temporary withdrawal of prednisolone.

Fifteen months later, there was no clinical, laboratory or whole body CT scan evidence of Hodgkin's disease. She remained neurologically well apart from slightly impaired manual dexterity and minor dysaesthesiae which were controlled by treatment with carbamazepine. Electrophysiological examination showed an increase in motor units to distal lower limb muscles which were previously partially denervated. 


\section{Discussion}

Clinically apparent peripheral neuropathy occurs in between $0.1 \%^{2}$ and $6 \%^{3}$ of cases of lymphoma, while electrophysiological evidence may be found in up to 20 to $40 \%$ of cases. ${ }^{4}$ Pathological mechanisms include direct infiltration of the nerve, which is rare $;^{6} 7$ demyelination; ${ }^{18}$ loss of dorsal root ganglion cells $;^{9}$ infection by herpes zoster $;{ }^{10}$ and an increased incidence of the Guillain-Barre syndrome. ${ }^{11}$ Several of the drugs used in the treatment of lymphoma, particularly vincristine, are neurotoxic ${ }^{12}$ and may adversely influence a pre-existing neuropathy. ${ }^{13}$

Polyneuropathies associated with carcinoma and lymphoma occur in several clinical forms which differ in their prognosis. Subacute sensory neuropathy ${ }^{1}$ is characterised by the progressive loss over a period of weeks of all sensory modalities in the extremities with relative preservation of motor power; the severe proprioceptive loss leads to disabling ataxia of gait. The spinal fluid protein is characteristically high. Pathologically, there is inflammation and degeneration of dorsal root ganglia whilst neurogenic muscular atrophy is said to be minimal.

The case described here fits well with the clinical features of this syndrome but is unusual in several respects. Firstly, this form of neuropathy is characteristically associated with bronchial carcinoma whereas its association with lymphoma is rare. Of 29 cases reviewed, ${ }^{1}$ one was associated with reticulum cell sarcoma and another with Hodgkin's disease but lymphomas were not otherwise seen amongst the associated neoplasms. Our patient had biopsyproven mixed cellularity Hodgkin's disease. Secondly, sensory neuropathy is said to carry a poor prognosis. None of the cases described by Horwich et al ${ }^{1}$ improved on treatment which included surgery, steroids, radiotherapy and cytotoxic agents. Our patient improved dramatically as the tumour was successfully treated even though this treatment included the known neurotoxic agents, vinblastine and procarbazine; she was able to return almost to normal life. The only other case described in association with Hodgkin's disease ${ }^{1}$ had no neurological improvement despite successful treatment of the underlying neoplasm. The tumour later recurred and, at autopsy, extensive irreversible cell loss, axonal loss and posterior column gliosis was found. Steroids were not given. In our case, the rapid time course of the improvement would suggest that the pathological mechanism involved had not extended to irreversible destruction of axons or dorsal root ganglia.

Subacute motor neuropathy has been described as a mirror image of subacute sensory neuropathy, but differs in being characteristically associated with lymphoma and in carrying a benign prognosis. ${ }^{8}$ Review of these two syndromes however reveals that both show some mixed features. Clinical or electrophysiological evidence of sensory abnormality, albeit mild, was found in eight of 10 patients with pure motor neuropathy. ${ }^{8}$ Similarly, mild weakness is mentioned in the clinical descriptions of three of seven patients with pure sensory neuropathy and in 12 of 26 cases reviewed from the literature $;^{1}$ the mild weakness of our case is of a similar degree. It may be that these "pure" neuropathies in fact merely represent ends of a continuous spectrum of mixed sensorimotor neuropathy in which the relative sensory or motor involvement is variable.

The good prognosis associated with our case resembles that of the lymphoma-associated motor neuropathies but contrasts with the uniformly poor prognosis reported in sensory neuropathy. ${ }^{1} \mathrm{We}$ suggest that the prognosis of the neuropathy is related more to the nature of the underlying tumour than the neuropathy and that successful treatment of the tumour may reverse the neurological changes of sensory as well as motor neuropathy.

We thank Dr JM Oxbury for permission to report this case and Mrs E Ward for the preparation of the manuscript.

\section{References}

${ }^{1}$ Horwich MS, Cho L, Porro RS, Posner JB. Subacute sensory neuropathy: a remote effect of carcinoma. Ann Neurol 1977;2:7-19.

2 Williams HM, Diamond HD, Craver LF, Parsons $\mathbf{H}$. Neurological complications of lymphomas and leukaemias. Springfield: Thomas, 1959.

3 Paul T, Katiyar BC, Misra S, Pant GC. Carcinomatous neuromuscular syndromes. Brain 1978;101:53-63.

4 Walsh JC. Neuropathy associated with lymphoma. $J$ Neurol Neurosurg Psychiatry 1971;34:42-50.

${ }^{5}$ Chaco J. Subclinical involvement of peripheral nerves in Hodgkin's disease. Oncology 1973;27:105-7.

${ }^{6}$ Guberman A, Rosenbaum H, Braciale T, Schlaepfer WW. Human neurolymphomatosis. J Neurol Sci 1978;36:1-12.

${ }^{7}$ Reid AC, Bone I. Lymphoma presenting as mononeuritis multiplex. Postgrad Med J 1981 ;57:176-7.

${ }^{8}$ Schold SC, Cho E-S, Somasundaram M, Posner JB. Subacute motor neuronopathy: a remote effect of lymphoma. Arn Neurol 1979;5:271-87.

${ }^{9}$ Sterman AB, Schaumberg HH, Asbury AK. The acute sensory neuropathy syndrome: a distinct clinical entity. Ann Neurol 1980;7:354-8.

${ }^{10}$ Ramella B. Les paralysies de nerfs périphériques 
d'origine zosterienne. Thesis 1965, Marseille.

${ }^{11}$ Currie S, Henson RA, Morgan HG, Poole AJ. The incidence of the non-metastatic neurological syndromes of obscure origin in the reticuloses. Brain $1970 ; 93: 629-40$.
${ }^{12}$ Weiss HD, Walker MD, Wiernik PH. Neurotoxicity of commonly used antineoplastic agents. New Eng $J$ Med 1974;291:127-33.

${ }^{13}$ Weiden BL, Wright SE. Vincristine neurotoxicity. New Eng J Med 1972;286:1369-70. 